\title{
Alternative In Vitro Methods to Characterize the Role of Endocrine Active Substances (EASs) in Hormone-Targeted Tissues
}

Istituto Superiore di Sanità, Rome, Italy, December 17, 2012

\author{
Costanza Rovida ${ }^{1}$, Isabella De Angelis ${ }^{2}$, and Stefano Lorenzetti ${ }^{2}$ \\ ${ }^{1}$ Center for Alternatives to Animal Testing (CAAT) - Europe, University of Konstanz, Germany; \\ ${ }^{2}$ Istituto Superiore di Sanità (ISS), Rome, Italy
}

\section{Introduction}

The CAAT-IPAM-ISS Symposium on "Alternative in vitro methods to characterize the role of Endocrine Active Substances (EASs) in hormone-targeted tissues" was jointly organized by the Istituto Superiore di Sanità (ISS), i.e., the Italian Governmental Health Institute, the Italian Platform for Alternative Methods (IPAM), central to the European Consensus-Platform for Alternatives (ecopa), and the European Center for Alternatives to Animal Testing (CAAT-Europe). About 60 participants attended the symposium, which was held in Rome, Italy at the ISS on December $17^{\text {th }}, 2012$.

The aim of the symposium was to emphasize the role of alternative methods in the evaluation of potential EASs through an open discussion between invited speakers and attendees. EASs represent a controversial issue, as they are perceived differently by scientists, regulators, and various stakeholders. Whereas some relevant in vitro assays have either been validated (e.g., on ER $\alpha$ transactivation) or are on the path of validation, the role of in vitro tests in a consistent risk assessment strategy on EASs has yet to be determined. Nevertheless, the three main EU regulations in force on chemicals (REACH, Regulation 2006/1907/EC), biocides (PPPs, Regulation 2009/1107/EC), and cosmetic products (Regulation 2009/1223/EC) strongly recommended the use of in vitro alternative methods, at least as a prioritizing screening approach to identify endocrine disrupting properties of EASs.

The area of EASs and Endocrine Disruptors (EDs) (i.e., EASs causing adverse effects mediated by endocrine mechanisms) has made scientific contributions leading to many publications. A PubMed search for the keyword "Endocrine Disruptor" returns 3997 articles until the end of 2012: 40\% of all publications were issued in the last three years, demonstrating that interest in this topic is still increasing. In spite of the enormous work that is focused on EDs, the scientific community is still divided, and there is no consensus on ED risk assessment because, according to some authors, EASs are supposed to challenge the canonical paradigms of toxicology, e.g., linear versus non-monotonic dose response, low dose effects versus threshold concept.

An overview of the symposium is given here; authorized presentations and the abstract book are available on the websites of the three organizing institutes, i.e., ISS (http://www.iss.it/inte/ prog/cont.php?id=263\&lang=2\&tipo=10); IPAM (http://www. ipamitalia.it), and CAAT (http://caat.jhsph.edu/media/slides/ed/ index.html).

\section{Introductory Session}

This session was chaired by Isabella De Angelis and Stefano Lorenzetti.

Thomas Hartung, in his presentation entitled Endocrine disruption as the pilot of mapping the human toxome, explained why animal models do not fit the purpose for the evaluation of endocrine disruption and illustrated an in vitro approach, a molecular means for the definition of Pathways of Toxicity (PoT) following the recent paradigm explained by the 2007 NAS/ NRC report: "Toxicity Testing in the $21^{\text {st }}$ Century: A Vision and a Strategy" (Tox21c, http://www.ncbi.nlm.nih.gov/pmc/articles/ PMC3202604/?tool=pubmed), that points out the need to switch from current in vivo testing to "... new tools, including functional genomics, proteomics, metabolomics, high-throughput screening, and systems biology, allowing us to replace current toxicology assays with tests that incorporate the mechanistic underpinnings of disease and of the underlying toxic side effects." Note that the NIH Human Toxome Project, led by Dr Hartung, uses estrogenic EASs as a pilot for developing the concept of PoT and their annotation.

Costanza Rovida's talk on Implementation of regulatory issues focused on the requirements of the EU Regulations, highlighting the many contradictions between the different regula- 
tions (Chemicals, Biocides, Cosmetics, Food Supplements, and Additives). Indeed, each regulation asks for an assessment of ED effects, but a clear and harmonized guideline on the use of in vitro data for the evaluation of endocrine disrupting activities is not yet available. This is especially important considering that the ED concerns should not be limited to the reproductive issue.

Alberto Mantovani, speaking on Endocrine Active Substances/EASs: understanding modes of action for risk assessment, discussed the scientific explanation for many common concepts that should be overlapped among different fields, e.g., in toxicology and risk assessment versus endocrinology. For instance, endogenous hormones and some vitamins surely are EASs, but at the same time they are essential, though at low doses, for the physiological growth and development of human beings. Due to their common mechanism of action (MoA), man-made EASs could mimic the role of the "natural" molecules, potentially competing with them. Furthermore, some toxicologists consider endocrine disruption as an endpoint per se but, depending on the target organ/tissue/cell, the proper endpoint should be identified case by case, e.g., EASs acting on the thyroid may lead to impaired neurobehavioral development. Taking into account each EAS' MoA(s), overlooked or unexpected effects (e.g., relevant to metabolic syndrome predisposition) should lead to the identification of novel cellular or molecular endpoints to be used as biomarkers. Finally, he warned the audience about two possible dangers that must be avoided: i) sticking to current toxicological approaches and ii) drowning in complexity.

The speaker from the European Food Safety Authority (EFSA), Johann Steinkellner, who addressed Exploration of alternative methods for toxicity assessment of pesticide metabolites, showed the importance of the identification of metabolite residues of a specific group of EASs, plant protection products, and, hence, of the impact of metabolic and degradation processes on their toxicological properties in food commodities. He also highlighted the applicability of the concept of TTC (Threshold for Toxicological Concern) even if, due to several current uncertainties, caution is recommended for EASs. Also, the engagement of EFSA in analyzing the opportunities for applying QSAR approaches to risk assessment is worth noting.

Serena Cinelli spoke about Improving test methods in the spirit of the 3Rs; the point of view of a contract research organization. She explained how the extensive experience of a CRO might provide support in the decision for the best testing strategy. However, CROs do not feel comfortable in proposing in vitro methods, as they generally are still expensive, especially when using omicsbased tools, and regulatory acceptance currently is uncertain. Regulators consider stand-alone in vitro methods insufficient for predicting in vivo effects; meanwhile, in vivo methods are accepted as such, even if they have not been formally validated and the relevance of the results has not been ascertained.

\section{Session 1: EASs in Reproductive Target Issues}

Session 1 was chaired by Simonetta Gemma and Marcello Spanò, who focused on the consequences that EASs may have on the reproductive system.
Stefano Lorenzetti, in his presentation A prostate perspective on male fertility and EASs: from toxicogenomics to phenotypic anchoring, discussed the prostate as an overlooked, independent target of EASs and the PSA (Prostate-Specific Antigen) secretion assay as a tool to investigate prostate-mediated effects on male reproduction. Overall, he pointed out the importance of using clinically relevant, functional biomarkers applied to human cells, both as a means of having a phenotypic anchoring to gene expression changes (i.e., toxicogenomics), and as a short-cut to bypass some relevant differences between human and rodent male reproductive systems, affecting the possible MoA of EASs. Finally, he also showed some preliminary data on the assessment of the "real" intracellular concentration of the tested chemicals versus the nominal one, indicating how various EASs have different intracellular bioavailability and distribution within subcellular compartments.

Marcello Spanò, speaking on Human sperm (epi)genetic biomarkers to assess the impact of EASs on male reproductive function, confirmed that infertility is a common problem of increasing incidence, together with other interrelated diseases such as testis cancer. This situation points to the urgent need for developing an assay to address the effects of EASs on human sperm. He also pointed out the importance of applying epigenetic approaches to reproductive toxicology: indeed, spermatogenesis could be considered both a reproductive and a developmental target, since effects of EASs on sperm (as well on other reproductive tissues) frequently are due to in utero exposure and might even be transferred to the next generation through epigenetic mechanisms.

In vitro effects of EASs in human placenta were then analyzed by Luana Paulesu, who spoke about the effect of environmental pollutants on human trophoblast-like cells. These highly specialized placental cells mediate the maternal-fetal interactions; whereas the critical role of endocrine regulation in pregnancy is well known, until recently, the placenta was considered only as a means for fetal exposure rather than being seen, itself, as a functional target of EAS. Some chemicals are known to interfere with trophoblast cells, but the full spectrum of EAS interactions with placental functions needs to be further investigated. In vitro methods based on human cells/tissues are particularly welcome in this field, due to the high degree of species specificity of the placental system, which means the animal model does not perfectly fit the purpose, and thus the development of assays based on human cells has to be endorsed.

\section{Session 2: EASs in different hormone-targeted tissues}

It is now well recognized that the effect of EASs should not be limited to the reproductive apparatus. That was the focus of Session 2, chaired by Alberto Mantovani and Paolo Marzullo.

In his presentation entitled Endocrine active nutrients explored in human-bone cell cultures Igor Bendik-Falconnier shared his experience in the area of nutraceuticals using human-bone cell cultures as a tool to assess efficacy and safety. Some EASs occur naturally in foods, representing fundamental ingredients of our diet. However, within nutraceuticals, such molecules are ad- 
ministered at much higher concentrations in comparison to the natural occurrence in food matrices. Bone metabolism is a wellknown target of physiological hormones, including sex steroids and thyroid hormones, as well as growth hormones. In this area, the action of natural bioactive substances (e.g., flavonoids) has received much more attention than xenobiotics. Although fulfilling important physiological roles, nutraceuticals acting as EASs must be carefully assessed, and the use of human bone cells in vitro may greatly support the risk-to-benefit assessment by addressing concentration-response relationships and the safety/efficacy balance.

Some considerations about potential neuronal response to EASs were presented by Robert A. Smith in The use of cell models in determining neuronal responses to EASs. The nervous system is highly complex and variable across both age and individual. Generally, hormones responsible for brain development are hardly ever considered. As for other tissues, since continuous cell lines are derived from tumors, they may lack important phenotypic features. For this reason, primary cell lines should be the preferred choice, but human-derived ones are almost unavailable. Also, the research on $\operatorname{MoA}(\mathrm{s})$ in the nervous system is lagging behind, with only some functional markers of the thyroid gland known to be specifically related to interaction with hormones and nuclear receptors and almost nothing else.

A very interesting approach may allow scaling down the toxicity to the digestive system by mimicking in vitro the metabolism and distribution of a chemical in an apparatus composed by connected devices containing cultures derived from different tissues. This advanced engineered solution was presented in the talk Dynamic in vitro organ models of metabolism by Arti Ahluwalia, who explained how this approach can be applied in practice, with interesting applications in the investigation of obesity as well. Such a system may be stable for a week, even if the response is generally obtained within minutes from contact with the test item.

\section{Session 3: EASs and kinetics}

In spite of its importance in the biological processes, kinetics is very often disregarded. Kinetics is particularly relevant in the definition of EDs, as it was explained during the Session 3, chaired by Thomas Hartung and Emanuela Testai.

Emanuela Testai, speaking about The role of biokinetics in in vitro tests and in the interpretation of results, highlighted that bioavailability and kinetics of toxicologically relevant molecules are at the basis of any toxicological evaluation, but it is even more relevant for in vitro assays where kinetics is usually indicated as responsible for in vitro/in vivo discrepancies. Despite this, the nominal (applied) concentration, rather than the active intracellular level of cell exposure, generally is associated with the observed effect. This concept, and the need to integrate kinetics and dynamics into the interpretation of in vitro data, was exemplified by results coming from an EU-funded project (Predict-IV). In the area of EDs, kinetics could be one of the causes for obtaining apparent non-monotonic dose-response, one of the controversial issues debated by many scientists.
The same concept was supported by Frédéric Yves Bois in his talk entitled Physiologically-based modeling of ovarian steroid hormones synthesis for EAS' health risk assessment. He explained that weighting the cell response with the real exposure may make an important difference. Generally, the relationship between dose and effect is assessed by animal models, but we know that it always contains some inherent inaccuracies and might even be misleading with regard to EASs.

Speaking on EASs contra human \& environmental health: Relevant or playground for merchants of doom?, Daniel R. Dietrich discussed the problem of EASs from a different perspective. Unfortunately, the opinion of the general public is biased by emotion and a politically-driven association between an effect and a possible exposure. Epidemiological data are valuable, but only if well designed and documented, with large enough cohorts. The same may be true with animal models that often are not statistically robust and that test unrealistically high dose levels - not to mention the biological differences between rodents and humans. However, it cannot be overlooked that rodent studies, notwithstanding their limitations, are the only information available for hazard characterization of many substances. Thus, it is necessary that novel in vitro testing approaches provide information that can be used for risk assessment.

\section{Discussion and conclusion}

The CAAT-IPAM-ISS Symposium ended with a lively discussion, despite the many presentations and the late hour, confirming that the interest in EASs is very high.

The main point of discussion concerned the limitations of the actual animal models, even though they still represent the only possibility that currently is fully accepted by regulators. Therefore, it is very important that scientists work in tight collaboration with regulators to design a fit-for-purpose integrated testing strategy that is scientifically sound as well as robust and efficient for regulatory purposes. From their side, regulators should create a common framework across the different areas of application (e.g., chemicals, biocides/plant protection products, cosmetics, food supplements or additives, etc.).

Overall, the critical open question remains the exposure to EASs: we live in a dynamic environment full of chemicals - either of natural or man-made sources - and thus, we should develop a full set of appropriate tools to assess the potential longterm effects of combined exposures to different EASs through the living environment and diet.

\section{Correspondence to}

Costanza Rovida

Center for Alternatives to Animal Testing - Europe

(CAAT-Europe)

University of Konstanz, POB 600

78457 Konstanz

Germany

e-mail: costanza.rovida@chimici.it 